\title{
Congestion Control Technique for Wireless Networks
}

\author{
Alhamali Masoud Alfrgani .Ali, Raghav Yadav, Hari Mohan Singh \\ Department of Computer Sciences\& Information Technology \\ 'Sam Higginbotom Institute of Agriculture, Technology \& Sciences Allahabad India'
}

\begin{abstract}
Congestion control in wireless networks has been extensively investigated over the years and several schemes and techniques have been developed, all with the aim of improving performance in wireless net-work. With the rapid expansion and implementation of wireless technology it is essential that the congestion control problem be solved. This paper presents a congestion control schemes which are different in slow start threshold calculation, bandwidth estimation, and congestion window manipulation. A comprehensive comparison of these approaches is given in relation to assumptions, bandwidth estimation, congestion window size manipulation, performance evaluation, fairness and friendliness and improved throughput.
\end{abstract}

\section{Introduction}

The congestion control in wireless networks has been extensively investigated over the years and several schemes and techniques have been developed, all with the aim of improving performance in wireless network. With the rapid expansion and implementation of wireless technology it is essential that the congestion control problem be solved. The Transmission Control Protocol (TCP) has been widely used in today's Internet. The protocol supports reliable data transport by establishing a connection between the transmitting and receiving ends. The transmitter starts a timeout mechanism when sending a packet to the receiver. The transmitter constantly tracks the round-trip times (RTTs) for its packets as a means to determine the appropriate timeout period. At the receiver, each received packet is acknowledged implicitly or explicitly to the transmitter. If the transmitter does not receive an acknowledgment for a given packet when the corresponding timeout period expires, the packet is deemed to be lost and subject to retransmission. A congestion window with dynamically adjusted size is used by the protocol to regulate the traffic flow from the transmitter to the receiver.

Although TCP was initially designed and optimized for wired networks, the growing popularity of wireless data applications has lead third generation wireless networks such as CDMA2000 and UMTS networks to extend TCP to wireless communications as well. The initial objective of TCP was to efficiently use the available bandwidth in the network and to avoid overloading the network (and the resulting packet losses) by appropriately throttling the senders' transmission rates. Network congestion is deemed to be the underlying reason for packet losses. Consequently, TCP performance is often unsatisfactory when used in wireless networks and requires various improvement techniques. A key factor causing the unsatisfactory performance is that the radio link quality in wireless networks can fluctuate greatly in time due to channel fading and user mobility

\subsection{Problems of TCP in Wireless Scenario}

TCP protocol may experience performance degradations over wireless networks, due to non-congestion related packet loss and varying round trip times Unmodified standard TCP performs poorly in a wireless environment due to its inability to distinguish packet losses caused by network congestion from those attributed to transmission errors. Packet loss or reception of out of- order packets indicates failures. To remove such failures ,TCP implements flow control and congestion control algorithms based on the sliding window. The performance of TCP is generally lower in wireless networks than in fixed.Thesis explained by the fact that TCP cannot distinguish problems that typically occur in wireless networks from congestion. The congestion control algorithms in TCP are based on the assumptions that data is lost mainly due to congestion and that data loss duet transmission errors is rare. Therefore, data loss is interpreted as a signal of congestion in the network. Even in a wireless network, where data loss may not be related to congestion, TCP considers it as a congestion case.TCP segments may be lost if the radio conditions are poor and the link layer protocol provides a low reliability. After some retransmission attempts the link layer protocol gives up and leaves further error recovery to TCP. Handover events may also lead to data loss. A whole window of data may be lost due to handover. Data loss due to an unreliable link layer or a handover may cause a timeout event followed by slow start or three Daypack followed by fast retransmit and fast recovery. In either case, the congestion control action taken by TCP is unnecessary. Directly after the loss event ,the radio quality may become high again, and after handover data may be transmitted without problems to the new base station.TCP may also misinterpret a sudden increase in the round trip time as data loss. If the delay is long enough for the retransmission timer to expire before an acknowledgments received, then TCP misinterprets the delay as an indication of data loss due to congestion. 
The delayed data is unnecessarily retransmitted and TCP enters slow start.A highly variable round trip time can also lead to a large RTO, since the RTO is based both on estimates of the round trip time and on variations in the round trip time. If the RTO is large, then TCP reacts slowly to data loss. Variations in the round trip time can be caused by link level retransmissions of a wireless link. If the link layer frames that contain a TCP segment must be retransmitted because of a poor radio environment, then the whole segment is delayed. Round trip time variations may also be caused by handover or competing traffic. Queuing in routers, base stations, and other intermediate nodes may also lead to a long round trip time. A long round trip time may cause low throughput and underutilization of the network, since it takes a number of round trip times before the congestion window reaches the capacity of the network. TCP performance is degraded, especially for short lived flows, which transmits a small amount of data.

\subsection{Congestion Control}

Window Based Congestion Control: In Window based congestion control, the sender window size is determined by the available buffer size in receiver (rwnd).In other words, we assumed that this is only the receiver that can dictate to the sender the size of sender window. We totally ignored another entity here -the network .If the network cannot deliver the data as fast as they are created by sender, it must tell the sender to slow down. In other word

The network is another entity that determines the size of sender's window. Sender window is also determined by the congestion in the network.

The actual size of window is minimum of these Actual window size =minimum (rwnd,cwnd)

\section{Rate Based Congestion Control}

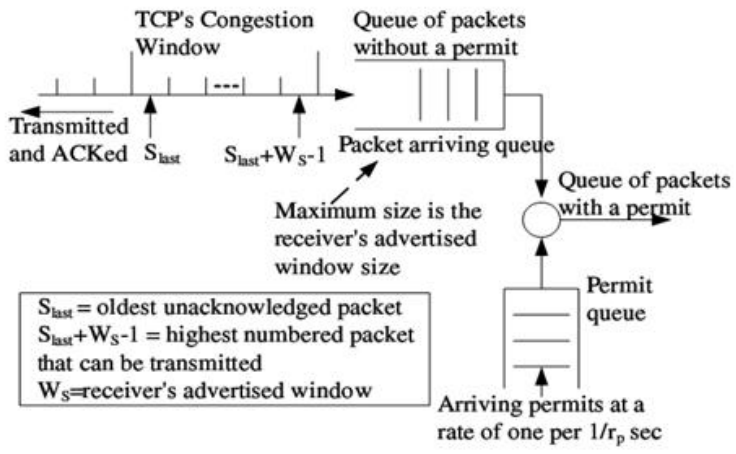

Fig. 1. Rate control mechanism

TCP's congestion avoidance mechanisms are not tuned for request-response traffic like HTTP. One such problem has to do with some TCP implementations forcing slow-start in the middle of a connection that has been idle for a certain amount of time, even if there is no packet loss. Other existing TCP implementations do not treat idle time as a special case and use the prior value of the congestion window to send data. It can lead to degradation of performance.

Rate based algorithms requires the following changes to TCP:

1. Idle time detection and indication that RBP needs to be started.

2. Bandwidth estimation.

3. Calculation of the window that we expect to send in RBP and the timing between segments in that window.

4. A mechanism that clocks the segments sent in RBP.

\section{Wireless Congestion Control Protocol (WCCP)}

TCP's congestion control suffers from a coarse granularity when applied to the multichip ad hoc environment. To overcome this problem, we propose a rate based wireless congestion control protocol (WCCP). There are two components in WCCP. One is at the transport layer. It replaces the window adjusting algorithm of TCP with a rate control algorithm to regulate the sending rate. The other is between the networking layer and the MAC layer. It monitors and possibly modifies the feedback field in TCP data packets when it passes the outgoing packets from the networking layer to the MAC layer and the incoming packets in the reverse direction.

In the rate-based congestion control algorithm, to calculate the ideal sending rate, the source is in dire need of a timely and easily measured metric which should satisfy two requirements. First, as mentioned in previous discussion, since MAC contention is tightly coupled with congestion, a candidate of congestion signal should reflect the condition of MAC contention and collision. Second, in order to fully utilize the shared channel 
without causing severe congestion and packet collision, the candidate should indicate the available bandwidth. The channel busyness ratio $r b$, which is defined as the ratio of time intervals when the channel is busy due to successful transmission or collision to the total time, meets these two requirements.

\section{Conclusion}

The proposed modification schemes to the standard TCP congestion control algorithm is better in their characteristics, algorithms and assumptions were presented. A comparison of their assumption, bandwidth estimation, window size manipulation, slow start phase, retransmission phases, congestion avoidance phase and performance evaluation methods is conducted. The need for an efficient method to optimally utilize available bandwidth is essential in the wireless links of combined wired and wireless networks. Future research is to evaluate these schemes against each other by comparing them in various network scenarios such varying bit error rates, number of connections, link capacity, and bandwidth.

\section{References}

[1]. Balakrishnan, H. and Padmanabhan, V. and Seshan, S. and Stemm, M. and Katz,R.: TCP behavior of a busy internet server: Analysis and improvements. Proceedingsof IEEE INFOCOM, 1998

[2]. Chandran, K. and Raghunathan, S. and Venkatesan, S. and Prakash, R.: AFeedback-based Scheme for Improving TCP Performance in Ad Hoc Wireless Networks.Proceedings of ICDCS, 1998

[3]. Chen, K. and Nahrstedt, K. and Vaidya, N.: The Utility of Explicit Rate-BasedFlow Control in Mobile Ad Hoc Networks. Proceedings of IEEE WCNC, 2004322 Y. Su and T. Gross

[4]. D. Kim, C.-K. Toh, and Y. Choi. "TCP-BuS: improving TCP performance in wireless ad hoc networks".journal ofcommunications and networks, vol. 3, no.2, june 2001.

[5]. Dina Katabi, Mark Handley, and Charlie Rohrs. "Congestion control for high bandwidth delay product networks."SIGCOMM COMPUT.COMMUN.REV.,32(4):89-102,2002.

[6]. Eckhardt, D. and Steenkiste, P.: Measurement and Analysis of the Error Characteristics of an In-Building Wireless Network. Proceedings of ACM SIGCOMM,1996

[7]. Fu, Z and Greenstein, B. and Meng, X. and Lu, S.: Design and Implementation of a TCP-Friendly Transport Protocol for Ad Hoc Wireless Networks. Proceedings of IEEE ICNP, 2002

[8]. Fu, Z. and Zerfos, P. and Luo, H. and Lu, S. and Zhang, L. and Gerla, M.: TheImpact of Multi-hop Wireless Channel on TCP Throughput and Loss. Proceedingsof IEEE INFOCOM, 2003

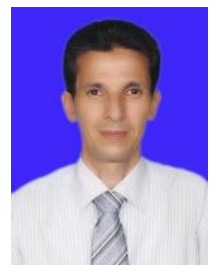

Alhamali Masoud Alfrgani .Ali ph.D student of SHIATS, B.tech degree in computer engineering from Technology College of Civil Aviation \& Meterology In year 1992 Tripoli Libya ,Mtech. From Sam Higginbotom Institute of Agriculture, Technology \& Sciences in year 2012.His research area is Computer net work

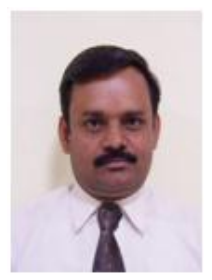

Raghav Yadav is obtain a ph.D degree from the Motilal Nehru National institute of technology (MNNIT),Allahabad india. He received his B.E degree in electronics engineering from Nagpur University and M.Tech degree in Computer Science and engineering from MNNIT.Allahabad. Mr Yadav is currently an assistant professor at Sam Higginbotom Institute of Agriculture, Technology \& Sciences (SHIATS). Allahabad, India .He has authored more than 10 research papers in national /international conferences and refereed journals .His research interests are in the field of optical net work survivability,ad-hoc net works, and fault tolerance systems.

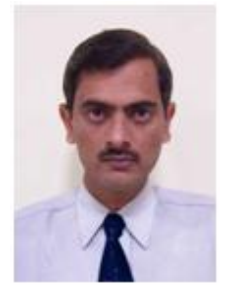

Hari Mohan Singh is presently pursuing a phD degree from MNNIT, Allahabad ,India, He received his B.F degree in computer science and engineering from Amravati University ,india.and M.Tech . degree in computer science and engineering from Uttar Pradesh Technical University (UPTU), Lucknow,india. Mr.Singh is currently an assistant professor at SHIATS, Allababad, india.

.He has authored more than eight research papers in national /international conferences and refereed journals .His research interests are in the field of optical net work survivability,realtime systems, and fault -tolerant systems 